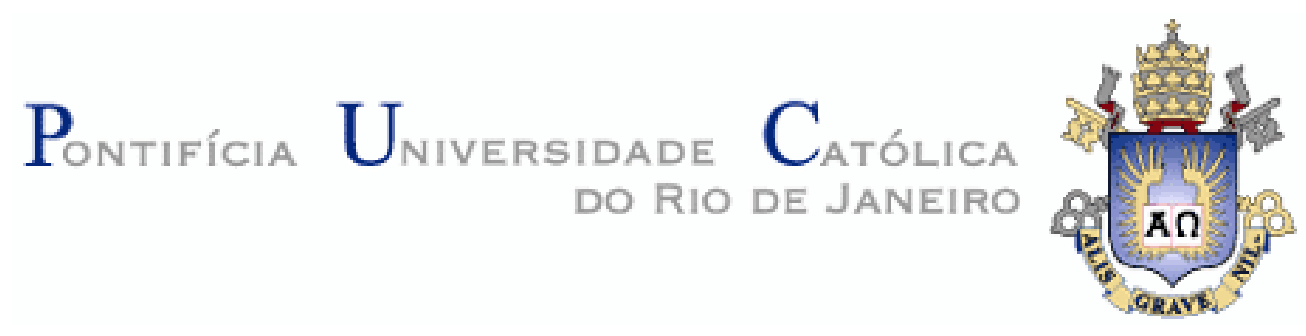

Vinci Pegoretti Amorim

Uma Arquitetura Flexível para Replicação de Bases Distribuídas Heterogêneas

Dissertação de Mestrado

Dissertação apresentada como requisito parcial para obtenção do título de Mestre pelo Programa de PósGraduação em Informática da PUC - Rio.

Orientador: Marco Antonio Casanova

Rio de Janeiro

Março de 2007 


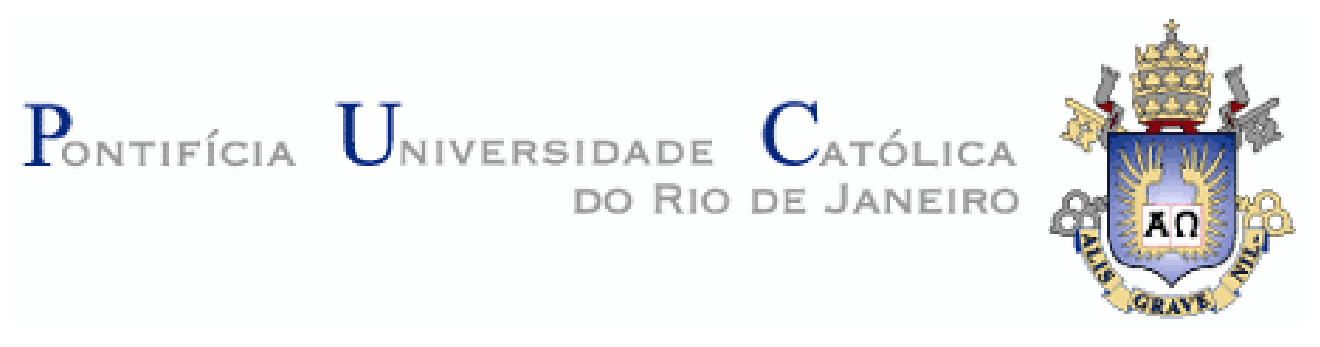

Vinci Pegoretti Amorim

\title{
Uma Arquitetura Flexível para Replicação de Bases \\ Distribuídas Heterogêneas
}

Dissertação apresentada como requisito parcial para obtenção do título de Mestre pelo Programa de Pós-Graduação em Informática da PUC - Rio. Aprovada pela Comissão Examinadora abaixo assinada.

\author{
Marco Antonio Casanova \\ Orientador \\ PUC - Rio \\ Antonio Luz Furtado \\ PUC - Rio \\ Karen K. Breitman \\ PUC - Rio \\ José Eugenio Leal \\ Coordenador Setorial do Centro Técnico Científico - PUC-Rio \\ Rio de Janeiro \\ Março de 2007
}


Todos os direitos reservados. É proibida a reprodução total ou parcial do trabalho sem autorização da universidade, do autor e do orientador.

\section{Vinci Pegoretti Amorim}

Graduou-se em Ciência da Computação na Universidade Federal de Viçosa - UFV 2004. Hoje é pesquisador no laboratório Tecgraf. Seus interesses incluem banco de dados, engenharia de software, inteligência artificial e sistemas distribuídos.

Ficha Catalográfica

Amorim, Vinci Pegoretti

Uma arquitetura flexível para replicação de bases distribuídas heterogêneas / Vinci Pegoretti Amorim ; orientador: Marco Antonio Casanova. - 2007.

60 f. ; $30 \mathrm{~cm}$

Dissertação (Mestrado em Informática)-Pontifícia Universidade Católica do Rio de Janeiro, Rio de Janeiro, 2007. Inclui bibliografia

1. Informática - Teses. 2. Replicação. 3. Bases heterogêneas. 4. Arquitetura. 5. Framework. 6. Agentes. 7. Arquitetura orientada a serviços. 8. InfoPAE. I. Casanova, Marco Antonio . II. Pontifícia Universidade Católica do Rio de Janeiro. Departamento de Informática. III. Título. 
Este trabalho é dedicado:

Ao meu orientador, por toda tranqüilidade e apoio acadêmico.

A meus colegas do Tecgraf, por toda camaradagem e apoio técnico.

À minha família, por toda confiança e apoio motivacional. 


\section{Agradecimentos}

Primeiramente gostaria de agradecer ao meu orientador, Marco Antônio Casanova, por explicar, facilitar e sugerir em seus devidos momentos.

Um agradecimento para os membros da banca de professores, Antonio Luz Furtado e Karen K. Breitman, que tão prontamente atenderam aos meus pedidos de participarem da avaliação desta dissertação.

Gostaria de agradecer aos colegas do laboratório Tecgraf, em especial ao Aldo Nogueira, Alexandre Gazola, Carlos Coutinho, Demétrius Nunes, Luis Gustavo Ferrão, Marcelo Tílio, Rafael March, Rodnei Couto, Roberto Santos, que estiveram envolvidos com diversos aspectos do desenvolvimento deste trabalho. O apoio de vocês foi vital.

Aos meus pais Rogelio Caetano Amorim e Regina Augusta Pegoretti Amorim e aos meus irmãos Rogelio Pegoretti Caetano Amorim e Darlon Pegoretti Amorim. Mesmo à distância, foram capazes de apoiar, motivar e confiar.

Aos amigos Gleidson, Victor, André, Nara e Anna Carina. 


\section{Resumo}

Amorim, Vinci; Casanova, Marco Antonio. Uma Arquitetura Flexível para Replicação de Bases Heterogêneas. Rio de Janeiro, 2007. 60p. Dissertação de Mestrado - Departamento de Informática, Pontifícia Universidade Católica do Rio de Janeiro.

Os diversos serviços de replicação existentes no mercado adquiriram um alto grau de maturidade e desempenho. Entretanto, mostram pouca capacidade de lidar com bases heterogêneas. Este trabalho propõe uma arquitetura focada na replicação de bases heterogêneas. Para conseguir alta escalabilidade e manter a simplicidade, a arquitetura segue uma estrutura multi-agente e adota uma abordagem de projeto orientado a domínio. Em seguida, o trabalho descreve uma implementação de referência e discute decisões técnicas desenvolvidas, enfatizando os problemas de versionamento, verificação de consistência e demais regras de negócio. O trabalho apresenta ainda utilitários que facilitam a configuração e manutenção do sistema de replicação.

\section{Palavras-chave}

Replicação; Bancos de Dados; Base Heterogênea; Agentes; Arquitetura Orientada a Serviços; Projeto Orientado a Domínio 


\section{Abstract}

Amorim, Vinci; Casanova, Marco Antonio. A Flexible Architecture for Replication of Heterogeneous Bases. Rio de Janeiro, 2007. 60p. MSc. Dissertation - Departamento de Informática, Pontifícia Universidade Católica do Rio de Janeiro.

The replication services available acquire high maturity and performance levels. However, they do not work with heterogeneous data bases. This dissertation first describes a software architecture that focus on how to provide replication services for heterogeneous bases. To obtain high scalability and to maintain simplicity, the architecture follows a multi-agent structure and adopts a domain-driven design approach. Then, the dissertation describes a reference implementation and discusses the technical decisions adopted, focusing on version control problems, consistency verification and specific businessoriented rules. The dissertation also describes utilities that facilitate the configuration and maintenance of the replication system.

\section{Keywords}

Replication; Heterogeneous Bases; Agents; Service Oriented Architecture; Domain-Driven Design 


\section{Sumário}

1 Introdução 12

1.1. Motivação 12

1.2. Objetivos 13

$\begin{array}{ll}\text { 1.3. Organização da Dissertação } & 13\end{array}$

2 Fundamentos 15

2.1. Replicação de Dados 15

2.2. Projeto Orientado a Domínio 16

$\begin{array}{lr}\text { 2.3. Software Orientado a Agentes } & 18\end{array}$

3 Arquitetura para Replicação de Bases Heterogêneas 20

3.1. Base Heterogênea 20

3.2. Serviço de Replicação de Bases Heterogêneas 21

3.2.1. Processamento em Ciclos 23

3.2.2. Versionamento 23

3.2.3. Controle dos Estados das Bases 24

3.2.4. Coordenação por Multi-Serviços de Replicação 25

3.2.5. Processamento Assíncrono 27

3.2.6. Operações Transacionais 27

3.3. Componentes da Arquitetura 27

3.3.1. Observador 29

3.3.2. Exportador 30

3.3.3. Importador 31

3.5.1. Configurador 32

3.5.2. Copiador 33

3.5.3. Sincronizador 34

3.5.4. Analisador 35

3.6. Agentes, Utilitários e Soluções 36

3.7. Camadas de um Serviço de Replicação 37 
3.7.1. Camada de Fronteira 38

3.7.2. Camada de Negócio 39

3.7.3. Camada de Coordenação 40

4 Um Exemplo de Implementação 41

4.1. InfoPAE 41

4.2. RePAE: Uma Implementação de Referência 43

4.3. Tecnologias Adotadas pelo RePAE 45

4.4. Componentes Físicos do Ambiente 47

4.5. Instalação e Configuração 48

4.6. Testes 51

5 Conclusão 53

5.1. Contribuições 53

5.2. Trabalhos Relacionados 53

5.2.1. SQL Server 54

5.2.2. Daffodil Replicator 54

5.2.3. Delta Replicator 54

5.3. Trabalhos Futuros $\quad 55$

6 Referências $\quad 57$

$\begin{array}{ll}\text { Apêndice A - Vocabulário } & 60\end{array}$ 


\section{Lista de figuras}

Figura 1. Estratégias de replicação de dados 16

$\begin{array}{lr}\text { Figura 2. Projeto orientado a domínio } & 18\end{array}$

Figura 3. Atuação de agentes em um ambiente 19

$\begin{array}{ll}\text { Figura 4. Base de dados heterogênea } & 20\end{array}$

$\begin{array}{ll}\text { Figura 5. Base de dados e serviço de replicação } & 21\end{array}$

Figura 6. Mapeamento entre os requisitos e as soluções 22

Figura 7. Possíveis trocas de estados de uma base $\quad 24$

Figura 8. Estratégias de coordenação de fluxo de replicação 25

Figura 9. Possíveis configurações de uma topologia com multi-serviços 26

Figura 10. Nó e topologia 26

Figura 11. Visão geral da arquitetura e utilitários 28

$\begin{array}{ll}\text { Figura 12. Driver observador } & 29\end{array}$

Figura 13. Exemplo de registro de alterações 30

Figura 14. Serviços de replicação 32

Figura 15. Utilitário Configurador 33

Figura 16. Utilitário copiador $\quad 34$

Figura 17. Utilitário sincronizador 34

Figura 18. Utilitário analisador 35

Figura 19. Mapeamento entre as soluções e os agentes e utilitários 37

Figura 20. Camadas $\quad 38$

Figura 21. Classes do pacote topologia $\quad 39$

Figura 22. Domínio do InfoPAE 42

Figura 23. Executando uma emergência no InfoPAE 43

Figura 24. Repositórios $\quad 44$

Figura 25. Base de dados do InfoPAE 44

Figura 26. Tecnologias 46

Figura 27. Exemplo de topologia lógica 47

Figura 28. Exemplo de topologia física 48

$\begin{array}{ll}\text { Figura 29. Estrutura de arquivos e diretórios } & 48\end{array}$ 
Figura 30. Exemplo de configuração de parâmetros 50

Figura 31. Configuração de uma topologia 51

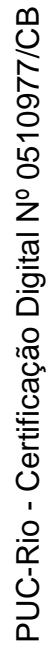

